\title{
Establishment and Clinical Application of an Electronic Database for Breast Cancer in China
}

\author{
Yong-Gang Lv, Mei-Ling Huang ${ }^{\&}$, Jing-Jing Xiao, Rui Ling*
}

\begin{abstract}
Purpose: To establish a database for breast cancer patients to save and manage clinical data and to preliminarily investigate its clinical application. Materials and Methods: Information on breast cancer patients hospitalized in our department from 2008.01 to 2013.01 were input into our breast cancer management system. SPSS 16.0 software was used as a convenient reference to evaluate the accuracy of the newly built database. Results: A database of $\mathbf{2 4 0 3}$ breast cancer patients was successfully established. Information in the database clearly displayed capabilities of storage, addition, retrieval, statistical analysis and other functions. As the continuously updated database showed, the distribution of age, sex, nationality, allergy history, pausimenia and marriage of patients was identical to that achieved by SPSS analysis, indicating reliable and accurate data analysis. Conclusions: The described database is easy and convenient to operate and manage, and should prove suitable for application in clinical research and treatment.
\end{abstract}

Keywords: Breast cancer - database - electronic management - China

Asian Pac J Cancer Prev, 17 (2), 639-641

\section{Introduction}

Breast cancer is now recognized as a publichealth issue on a global scale, which is responsible for $23 \%$ of total cancer cases and $14 \%$ of cancer deaths worldwide(Jansen et al., 2014). As the emergency of accurate therapy, enhanced understanding of breast cancer might be significant for better diagnosis and treatment. However, it is not convenient to consult and study these data during clinical research. The lack of systematic collection about the clinical date makes it difficult to interpret the mechanism and explore novel findings.

Electronic medical record (EMR), as traditional model of medical database, has been unable to meet the requirement of disease information review and analysis. As the development of computer technique, database technology achieved unprecedented development at present (Nigrin and Kohane, 2000; Lee et al., 2001), especially in medical area (Brown et al., 2001). Since 1997, a relational database used in the management of patients with retinoblastoma has been designed (Di Pisa et al., 1997). In 2008, Bayley, et al. built an online database of fumarate hydratase mutations involved in the multiple cutaneous and uterine leiomyomas (MCUL) tumor syndrome and congenital fumarase deficiency (Bayley et al., 2008). However, limited published multicenter data on practical and clinical changes in patients with breast cancer are available.
Since then, the computer technique was employed in the present study to establish the breast cancer management system, which makes the administration of patients' data more scientific, convenient, and effective than before. Here we presents the characteristics and information platform of included cases and the applicability of breast cancer database.

\section{Materials and Methods}

\section{Database}

The breast cancer database was integrated. For each case, there were 1439 items, including almost all information of patients, such as basic information like sex, marriage, age; pre-operative diagnosis; operation pattern; post-operative condition like TNM stage, complication; as well as the follow-up information. This system is characterized as follows: (1) Designed system is close to clinical reality, which is easy to use without any training course. (2) The network technique makes it possible to revise, delete and retrieve every item in database. There is a one-to-one correspondence between patient data and patients' identification number. (3) Established statistical function allows clinicians to easily observe the trend and distribution differences in the library at different stages, obtaining comprehensive knowledge of breast cancer patient included. (4) The export and backup function ensure the safety of database when system is up-grated. 


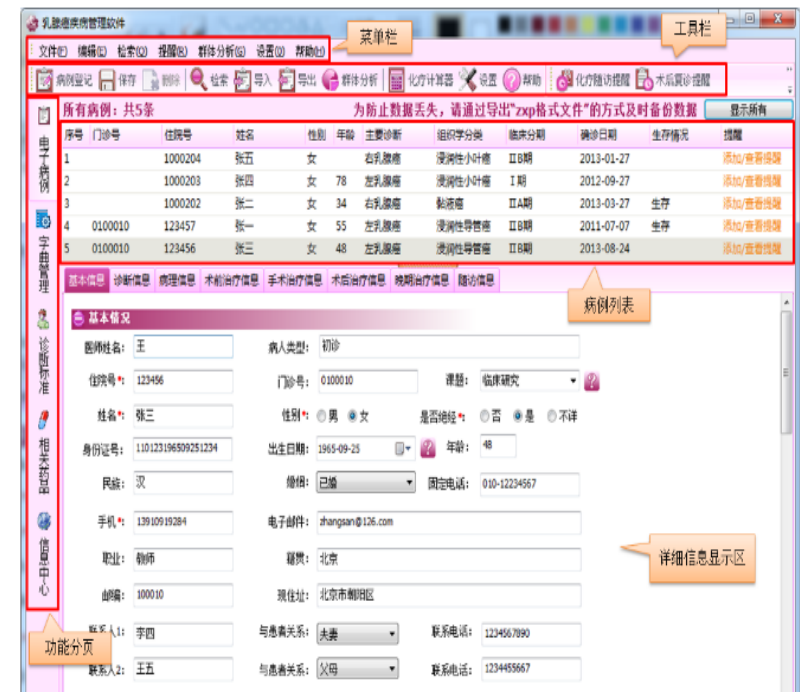

Figure 1. Main Screen for the Breast Cancer Database
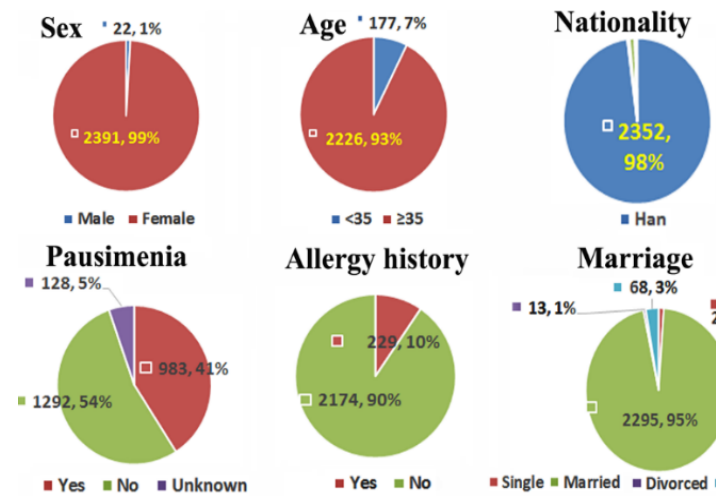

Allergy history

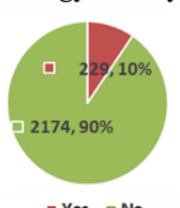

Marriage

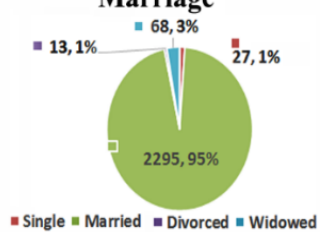

Figure 2. Statistical Analysis Results by Established Database

Table 1. Information Collected by SPSS 16.0 Software

\begin{tabular}{lc}
\hline Index & Statistics (N/\%) \\
\hline Sex & \\
Male & $22(1 \%)$ \\
Female & $2391(99 \%)$ \\
Age & \\
$<35$ & $177(7 \%)$ \\
$\geq 35$ & $2226(93 \%)$ \\
Mean & 48.9 \\
Median (range) & $48(15-86)$ \\
Nationality & \\
Han & $2352(98 \%)$ \\
Minortity & $51(2 \%)$ \\
Pausimenia & \\
Yes & $983(40.9 \%)$ \\
No & $1292(53.8 \%)$ \\
Unknown & $128(5.3 \%)$ \\
Allergy history & \\
Yes & $229(9.5 \%)$ \\
No & $2174(90.5 \%)$ \\
Marriage & \\
Single & $27(1.1 \%)$ \\
Married & $2295(95.5 \%)$ \\
Divorced & $13(0.6 \%)$ \\
Widowed & $68(2.8 \%)$ \\
\hline
\end{tabular}

\section{Database input and evaluation}

The clinical information of patients with diagnosed breast cancer admitted to the Xijing Hospital was input from 2009.01 to 2014.09. SPSS 16.0 was used to verify the accuracy of breast cancer database.

\section{Results}

Establishment of the anatomical ultrathin cross-section database

We established a breast cancer database containing 2403 cases, and > 20 different types, of fetal CHDs, including invasive ductal carcinoma (IDC), invasive lobular carcinoma (ILC), papillary carcinoma, carcinoma in situ, squamous-cell carcinoma, mucinous carcinoma, et al. Data input was simple and rapid, data query was convenient. In addition, data maintenance was safe and reliable. It could realize information based management of the cases.

\section{Statistical function of databases}

The database possessed the function of retrieval and statistical analysis, which is significant to clinica research, which is significant to clinical research. To measure the statistical accuracy of database, SPSS software was applied in this study. Compared with the SPSS statistics shown in Table 1, results achieved by database was in coordinate with SPSS outcomes, not only from the aspects of the percentage of female, but also from the proportion of age, nationality, pausimenia, allergy history and marriage (Figure 2), reflecting the practical and accuracy of established database.

\section{Discussion}

Breast cancer remains a public-health issue on a global scale(James et al., 2015). Establishing a cancer library was significant for clinicians to take a full advantage of clinical resources, powerfully supporting the extensive clinical research. Here we built a breast cancer database for case collection and claim the necessity of digital management for breast cancer patients.

Our database presents not only the overall information of each patient. The input was also convenient to operate and the database can be stored and easy to carry. Importantly, the searching function and statistical analysis can be proceeded according to the practical requirement. Export, analysis, save and backup guaranteed the safety of database. Up to date, the database has been proceeded perfectly under the regular maintenance of engineer. The included cases has been up to 2000 , which would be richer as the development of clinical treatment.

Recently, cancer database is been programmed according to the international standard. Nevertheless, similar database is rare to be found in China. The subjects and orientation are limited, with uneven quality. Our exploration demonstrated that the breast cancer database can be a useful clinical tool for collecting information of breast cancer patients. To promote the date share and combination, we appeal every hospital to establish breast cancer database. 


\section{Acknowledgements}

This operation was funded by National Science

Foundation of China (NO.5084681)

\section{References}

Bayley JP, Launonen V, Tomlinson IP (2008). The FH mutation database: an online database of fumarate hydratase mutations involved in the MCUL (HLRCC) tumor syndrome and congenital fumarase deficiency. BMC Med Genet, 9, 20.

Brown ML, Lipscomb J, Snyder C (2001). The burden of illness of cancer: economic cost and quality of life. Annu Rev Public Health, 22, 91-113.

Di Pisa F, Mastrangelo D, Hadjistilianou T, et al (1997). Design and implementation of a relational database used in the management of patients with retinoblastoma. Comput Biomed Res, 30, 273-89.

James FR, Wootton S, Jackson A, et al (2015). Obesity in breast cancer - What is the risk factor? Eur J Cancer, 51, 705-20.

Jansen LA, Backstein RM, Brown MH (2014). Breast size and breast cancer: A systematic review. J Plastic Reconstructive Aesthetic Surgery, 67, 1615-23.

Lee J, Kim J, Lee J, et al (2001). The study on the web-based Clinical Database Management System of Oriental Pulse Waveform. J Med Syst, 25, 277-84.

Nigrin DJ, Kohane IS (2000). Temporal expressiveness in querying a time-stamp--based clinical database. J Am Med Inform Assoc, 7, 152-63. 